\title{
Mit Softwaretools zu nachhaltigen Produkt- und Serviceinnovationen
}

\section{Unternehmen können durch Produkt- und Servi- ceinnovationen einen erheblichen Beitrag zu einer nachhaltigen Entwicklung leisten. Die erfolg- reiche Entwicklung von Innovationen ist aber nur durch die systematische Aufbereitung von Infor- mationen möglich. Softwaretools bieten dazu neue Möglichkeiten. Von Stefanie Springer, Severin Beucker, Daniel Heubach, Fabian Kaiser, Dierk-Oliver Kiehne und Mihály Jakob}

\footnotetext{
n der Entwicklung intelligenter und nachhaltigskeitsorientierter Produkt- und Serviceinnovationen liegt ein wichtiger Beitrag, den Unternehmen bei der Umsetzung einer nachhaltigen Informationsgesellschaft leisten könnten (Angrick 2003). Als Nachhaltigkeitsinnovation wird die Durchsetzung solcher technischer oder sozialer Neuerungen angesehen, die zum Erhalt kritischer Naturgüter und zu global und langfristig übertragbaren Wirtschafts- und Konsumstilen beitragen (Fichter/Noack et al. 2005).
}

\section{Innovationsmanagement in einer nachhaltigen Informationsgesellschaft}

Grundsätzlich wird das Verhältnis zwischen dem Innovationsbegriff und dem Leitbild der nachhaltigen Entwicklung alles andere als eindeutig bewertet (Fichter 2005). Auf der einen Seite stehen positive Betrachtungen, die das Leitbild der nachhaltigen Entwicklung als Ideengeber und Quelle von Wettbewerbsvorteilen ansehen oder aber auf einen erhöhten Innovationsdruck durch drohende Umweltprobleme hinweisen. Kritische Betrachter hingegen sehen in ökologischen und ethisch-sozialen Erwägungen ein potenzielles Innovationshemmnis.

Ebenso kontrovers wird die umgekehrte Wirkungsrichtung diskutiert, das heißt inwieweit das Bemühen um Innovationen positiv oder negativ für eine nachhaltige Entwicklung ist oder sein kann. Konsens besteht darin, dass zahlreiche, ehemals innovative Produkte die heutigen Umweltprobleme und das ressourcenintensive Konsumniveau westlicher Industrieländer erst verursacht haben. Innovationen leisten insofern nicht per se einen positiven Beitrag zu einer nachhaltigen Entwicklung, sondern sind in ein komplexes Wirkungsgeflecht eingebettet, in dem insbesondere indirekte und nichtintendierte Nebenfolgen beachtet werden müssen.
Dennoch stecken in innovativen Produkten, nicht zuletzt in der Verbindung mit informationstechnischen Elementen, viele Möglichkeiten, die Material- und Ressourcenproduktivität zu erhöhen. Neue Produkt-Systemlösungen auf digitaler Basis, die Funktionen von konkreten Produkten entkoppeln, können die Umsetzung des Nachhaltigkeitsprinzips unterstützen. Dennoch zeigt sich auch, dass die systemimmanenten Risiken technologischer Innovation genauso wie das Nutzerverhalten intensiver als bisher zu berücksichtigen sind, um konterkarierende Entwicklungen zu vermeiden (Paech/Pfriem 2004). Doch wie können Innovationsprozesse, die diese Wechselbeziehungen berücksichtigen, mithilfe des Internet unterstützt werden?

\section{Management früher Innovationsphasen}

Die Berücksichtigung nachhaltigkeitsrelevanter Informationen in sehr frühen Innovationsphasen kann die Nachhaltigkeitswirkung des Innovationsergebnisses positiv beeinflussen. Insgesamt stellen frühe Innovationsphasen für Unternehmen eine besondere Herausforderung dar. Neue Produkte und Dienstleistungen entstehen mittlerweile in einem Umfeld zunehmender Informationsverfügbarkeit und steigender Wissensintensivierung. Ihre Entstehung ist durch eine zeitliche und räumliche Entkoppelung und Virtualisierung des Innovationsund Entwicklungsprozesses gekennzeichnet. Steigende Informationsverfügbarkeit sowie kürzere Halbwertszeiten von Wissen, der zentralen Ressource für Innovationsprozesse, verstärken diesen Effekt (Fichter/Beucker 2006). Darüber hinaus müssen im Innovationsprozess zunehmend komplexe rechtliche und gesellschaftliche Rahmenbedingungen, wie beispielsweise Produkthaftung und Umweltschutzanforderungen berücksichtigt werden (Züst 1998). Parallel findet eine starke Dynamisierung betrieblicher Innovationsprozesse statt, kurze Marktzyklen und große Variantenvielfalt sind zwei Merkmale dieser Entwicklung. Aus dieser Ausgangssituation lassen sich unter anderem die folgenden Fragestellungen für den Innovationsprozess ableiten:

- Wie kann in frühen Innovationsphasen Orientierungswissen geschaffen und strukturiert werden, das bei einer Entscheidungsfindung über den Einstieg in ein nachhaltiges Technologie- oder Marktfeld oder auch ein bestimmtes Produktsegment unterstützt?

- Welche Informationsquellen können für den Aufbau des nachhaltigen Orientierungswissens hilfreich sein und wie kann die Aktualität und Qualität der Informationen gesichert werden? 
Das Verbundprojekt Nova-net beschäftigte sich von 2003 bis 2007 mit Innovation in der Internetökonomie und wurde durch das Bundesministerium für Bildung und Forschung im Programm Internetökonomie gefördert (1). Nova-net hat sich mit diesen zentralen Fragestellungen beschäftigt und mit dem delphigestützten Szenario-Management sowie der Expertensuche zwei softwaregestützte Methoden entwickelt, die Unternehmen bei der Beschaffung, Strukturierung und Bewertung von Informationen in frühen Innovationsphasen unterstützen.

\section{Delphigestütztes Szenario-Management}

Frühe Innovationsphasen sind durch einen hohen Bedarf an strukturierter Darstellung von Einflussgrößen für die Produktentwicklung gekennzeichnet (Müller-Stewens/Lechner 2001).

Bezogen auf das Leitbild der nachhaltigen Entwicklung ist es entscheidend, bereits zu einem frühen Zeitpunkt Abschätzungen über die Nachhaltigkeitswirkung einer Innovation zu integrieren. Dabei ist es zentral neben den unterschiedlichen Trends auch die Beachtung der Wechselwirkungen verschiedener und oft in einem dilemmatischen Verhältnis stehender Informationen sowie Nachhaltigkeitsanforderungen zu berücksichtigen. Die im Projekt Nova-net entwickelte Methode eines delphigestützten Szenario-Managements ermöglicht die Entwicklung strukturierter Zukunfts-Szenarien, mit deren Hilfe mögliche Fehlentscheidungen und deren Folgekosten vermieden werden können (Jakob et al. 2007). Folgende Schritte sind Bestandteil der Methode:

- Einflussanalyse: Zunächst werden die für das Technologieoder Geschäftsfeld relevanten Einflussfaktoren identifiziert und Zukunftsoptionen, die die verschiedenen möglichen Entwicklungsrichtungen zu diesem Einflussfaktor darstellen, ermittelt. Anschließend werden die relevanten Einflussfaktoren den strategischen Fragestellungen zugeordnet. Abbildung 1 zeigt die Zuordnung der Einflussfaktoren Ölpreis und Strompreis zur Beispielfragestellung nach den Erfolgschancen eines Personenkraftwagens mit Hybridtechnologie.

Abbildung 1: Strukturierte Szenariodarstellung

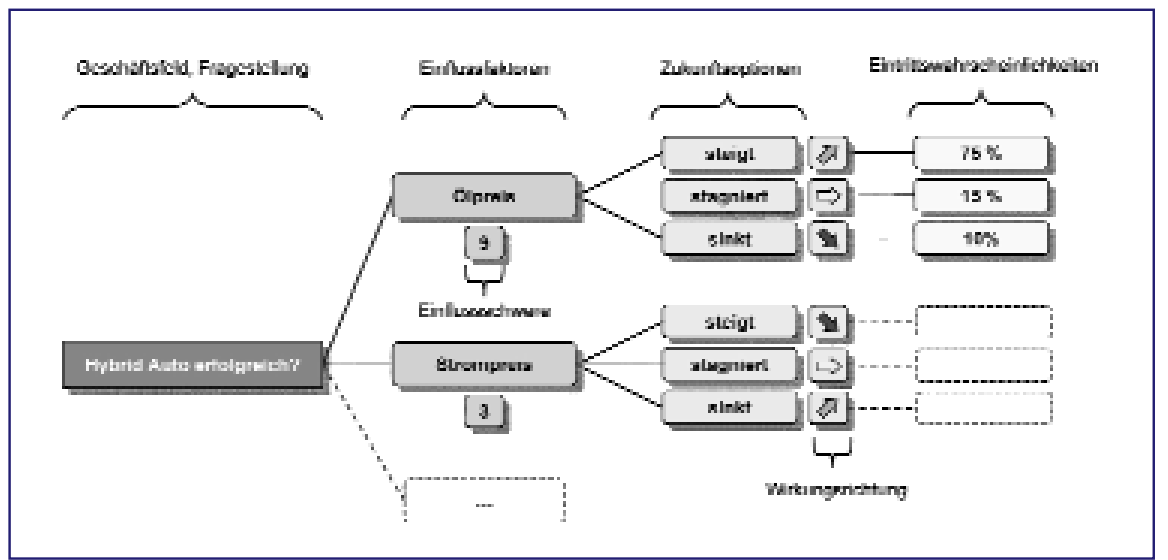

Quelle: Eigene Darstellung
- Wirkungsanalyse: Mit einem einfachen Bewertungsverfahren wird die Einflussschwere von Einflussfaktoren sowie deren Wirkungsrichtung beim Eintreten einer Zukunftsoption auf ein zu untersuchendes Geschäfts- oder Technologiefeld bewertet. Dadurch wird der Bezug der Einflussfaktoren zu den strategischen Fragestellungen weiter konkretisiert. Im aufgeführten Beispiel wird dem Einflussfaktor Ölpreis die Einflussschwere neun zugeordnet. Die zu diesem Einflussfaktor gehörenden Zukunftsoptionen „steigt“, „stagniert“ und „sinkt“ werden mit den Wirkungsrichtungen „positiv“, „neutral“ und „negativ“ bewertet.

- Bewertung der Wahrscheinlichkeiten: Im nächsten Schritt werden die Wahrscheinlichkeiten für das Eintreten von Zukunftsoptionen mittels einer delphibasierten Expertenbefragung über ein Internet-Formular bewertet. Dadurch wird es ermöglicht, Entwicklungen weitgehend manipulationsfrei einzuschätzen. Die Gesamtheit der Zukunftsoptionen mit den jeweils höchsten Eintrittswahrscheinlichkeiten repräsentiert das wahrscheinlichste Zukunftsszenario.

- Berechnung eines Indikators: Aus den Daten der Schritte eins und zwei wird ein Indikator berechnet. Dieser erlaubt eine Aussage darüber, wie gut ein Untersuchungsgegenstand, beispielsweise ein Technologie- oder Geschäftsfeld in das wahrscheinlichste Zukunftsszenario passt.

Zukunftsszenarien, die durch den Einsatz der vorgestellten Methode entstehen, können für die Einschätzung von Produktideen sowie deren Nachhaltigkeitswirkung verwendet werden. Damit ist die Methode sowohl für die Phase der Ideenakzeptierung als auch für die Phase der Ideenrealisierung relevant. In der erstgenannten Phase können für verschiedene Produktideen entsprechende Szenarien gebildet und miteinander verglichen werden. Dadurch ist der zukünftige Erfolg oder Misserfolg verschiedener Produkte sehr früh einschätzbar und die Auswahl der vielversprechenden Produktideen möglich. Zusätzlich besteht die Möglichkeit, in der Phase der Ideenrealisierung die Szenarienbildung für ausgewählte Produktideen periodisch zu wiederholen, um damit den prognostizierten Erfolg des entwickelten Produktes zu überwachen.

Für die Umsetzung der Methode wurde das modular erweiterbare, informationstechnische Framework Semafor entwickelt (Jakob et al. 2005). Die entstandene Web-Applikation ermöglicht sowohl die verteilte Entwicklung von Szenarien als auch die Durchführung von webbasierten Delphi-Befragungen von Experten, indem es die einzelnen Arbeitsschritte des delphigestützten Szenario-Managements unterstützt.

Allgemein spielt in den frühen Innovationsphasen, aber auch für die vorgestellte Methode des delphigestützten Szenariomanagements, Expertenwissen eine zentrale Rolle. Experten können dabei als 
Fachkundige beschrieben werden, die sich das Wissen über eine bestimmte Technologie oder ein Themenfeld angeeignet haben. Im Hinblick auf die Entwicklung von Nachhaltigkeitsinnovationen sind vor allem Experten mit nachhaltigkeitsrelevantem Wissen einzubinden.

Diese Experten finden sich sowohl innerhalb als auch außerhalb einer Organisation. Besonders für kleine und mittlere Unternehmen spielt externes Expertenwissen eine große Rolle, da diese meist nicht in der Lage sind, alle relevanten Technologie- und Anwendungsfelder mit internen Ressourcen abzudecken. Die Problematik bei der Identifikation von Experten ist, dass eine unstrukturierte Suche viel Zeit und Personalressourcen kosten kann und es oft kaum möglich ist, die Expertise der identifizierten Personen realistisch zu bewerten.

\section{Expertensuche im Internet}

Die Suche nach Experten untergliedert sich in zwei Teilaufgaben, die im Wechsel und wiederholt durchgeführt werden. Zum einen die Suche nach Personen beziehungsweise Institutionen, welche auf dem gesuchten Themengebiet Kompetenzen besitzen sowie zum anderen die Bewertung der Ergebnisse dieser Suche. Unterschiedliche Medien und Quellen können hierfür eingesetzt werden. Beispiele sind Branchensuchdienste, Patentdatenbanken, persönliche Kontakte oder das World Wide Web. Die hier vorgestellte Methode und Software fokussiert dabei auf das Internet mit seinen zum Teil öffentlich zugänglichen Daten. Sie nutzt dabei die stetig wachsende Informationsverfügbarkeit, um schnell und kostengünstig an externes Wissen zu gelangen und damit der Dynamisierung von Innovationsprozessen Rechnung zu tragen.

Neben den oben genannten Vorteilen des Internets bringt seine Nutzung auch Probleme mit sich. Eine manuelle Suche erfordert gute Fähigkeiten im Umgang mit Suchmaschinen, da beispielsweise eine präzise Anfrage die Voraussetzung für gute Suchergebnisse ist. Sie ist aufgrund der Informationsfülle des Internet zeit- und ressourcenaufwendig und damit oftmals teuer. Eine weitere Schwierigkeit besteht darin, dass im Internet verfügbare Daten meist nur schwach strukturiert sind, was eine schnelle Verarbeitung erschwert. Um aus diesen Daten verwertbare Informationen zu gewinnen, wird eine strukturierte Vorgehensweise benötigt, die dem Anwender einen Leitfaden für effizientes Suchen zur Verfügung stellt und ihn durch geeignete Softwaretools bei der Verarbeitung und der Analyse der anfallenden Datenmengen unterstützt.

\section{Vorgehensweise für die Expertensuche}

Die im Rahmen von Nova-net entwickelte Methode zur Suche nach Experten bietet dem Anwender einen dreistufigen Prozess, anhand dessen er die Suche strukturieren kann. Dieser Prozess ist in Abbildung 2 dargestellt. Mit der Software Expose, die als technische Umsetzung der Methode entwickelt wurde, erhält der Anwender zusätzlich ein Hilfsmittel, das den Prozess

\section{„Die Berücksichtigung nachhaltig- keitsrelevanter Informationen in frühen Innovationsphasen kann das Innovations- ergebnis positiv beeinflussen."}

weitgehend unterstützt (Kaiser et al. 2007).

Nachfolgend werden die einzelnen Schritte des Prozesses vorgestellt und die jeweilige Softwareunterstützung aufgezeigt. I Zuerst muss der Informationsbedarf spezifiziert werden. Die Präzision der Suchanfrage ist der beherrschende Einflussfaktor für die Qualität der Suchergebnisse. Die Herausforderung bei der Formulierung von Suchanfragen liegt in der Wahl der richtigen Suchbegriffe und deren logischer Verknüpfung. Dazu wird ausgehend von den Suchbegriffen des Anwenders ein umfangreiches Begriffsnetz aufgebaut, das unterschiedliche Aspekte des Themenfeldes sowie verwandte Themenkomplexe und den Bezug zwischen diesen darstellt. Die Softwareunterstützung in diesem Schritt besteht darin, die Generierung der Begriffsnetze weitgehend zu automatisieren. Dies geschieht durch Analyse der begrifflichen Beziehung in großen Text- und Strukturdatenbanken wie beispielsweise Wikipedia. Innerhalb dieses Begriffsnetzes können nun relevante und irrelevante Bereiche vom Anwender identifiziert werden und die daraus gewonnenen Erkenntnisse im folgenden Schritt bei der Suche nach relevanten Ressourcen genutzt werden.

I Im zweiten Schritt muss nach passenden Dokumenten gesucht werden. Anhand der Suchspezifikation aus Schritt eins erfolgt nun eine Suche im Internet. Dabei werden verschiedene Suchmethoden verfolgt. Neben der Suche mittels herkömmlicher Suchmaschinen im öffentlich zugänglichen Web werden ebenso spezielle Netzwerke, Forschungsein- $\rightarrow$

Abbildung 2: Der Suchprozess mit EXPOSE

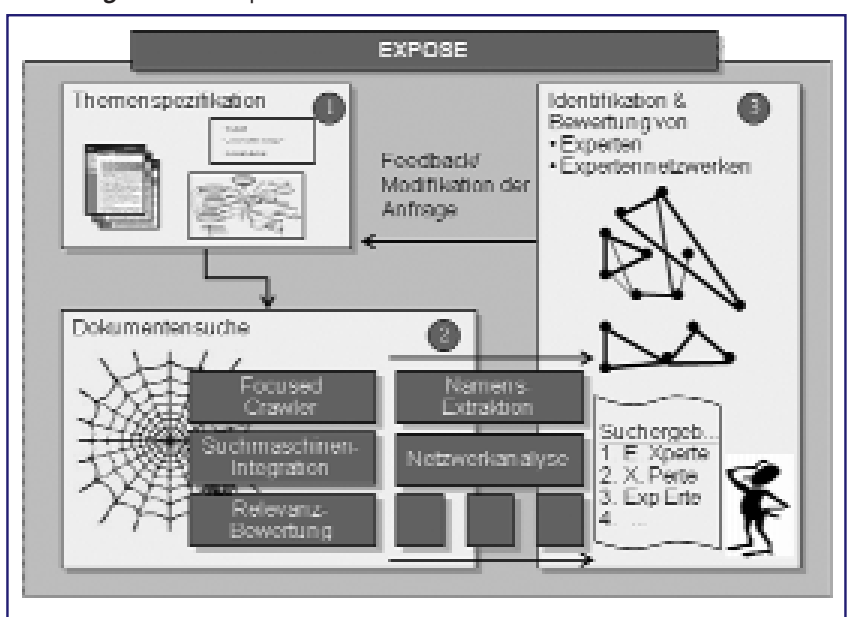

Quelle: Eigene Darstellung 
richtungen, Datenbanken und Ähnliches durchsucht. Dies kann sowohl manuell erfolgen als auch unterstützt durch Expose, indem Anfragen automatisiert generiert und ausgewertet werden und das Umfeld vielversprechender Dokumente näher untersucht wird. Alle Suchergebnisse sowie weitere Informationen über den Suchstrang, die Bewertung und strukturelle Zusammenhänge werden protokolliert und für die Auswertung im folgenden Schritt gespeichert.

I Im dritten Schritt erfolgt die Identifikation und Bewertung von Experten. Die in den vorangegangenen Phasen gesammelten Daten werden dabei ausgewertet. Das Resultat ist eine Liste mit Personen oder Institutionen, die vom System als besonders kompetent auf dem spezifizierten Themenfeld angesehen werden. Die Einschätzung basiert auf Kennzahlen und Kriterien, die automatisch aus den gesammelten Daten berechnet und vom Anwender situationsabhängig gewichtet werden können. Beispiele sind Textähnlichkeiten, Vorkommen und Bezug kennzeichnender Begriffe, Quelle der Nennung, netztopologische Zusammenhänge oder Beziehungen zwischen den identifizierten Personen.

Die softwaregestützte Methode wurde entwickelt, um dem Anwender einen Leitfaden und die entsprechende softwaretechnische Unterstützung zur Suche nach Experten im Internet an die Hand zu geben. Aufgrund verschiedener Unsicherheiten und der nur bedingt abschätzbaren Qualität von Webressourcen kann Expose naturgemäß keine definitive Aussage über die Expertise der identifizierten Personen machen. Um jedoch den Einfluss von Fehlern möglichst gering zu halten, bietet die Software dem Benutzer verschiedene Möglichkeiten. Beispielsweise können häufig fehlerhaft erkannte Namen über eine Mustererkennung von der Bewertung ausgeschlossen, es können Zugaben oder Abzüge in der Bewertung von bestimmten Domains oder Personen vergeben werden und vieles mehr. Dennoch wird ein derartiges System immer eine gewisse Fehlerquote aufweisen. Diese möglichst gering zu halten und den Benutzer bei der Auswahl seiner Experten zu unterstützen ist das Ziel von Expose.

\section{Fazit}

Die im Forschungsprojekt Nova-net entwickelten Vorgehensweisen für ein delphibasiertes Szenariomanagement sowie die Expertensuche können nachhaltige Produkt- und Serviceinnovation durch die Aufbereitung von relevanten Informationen in der Entscheidungsfindung unterstützen. Ziel ist es, Suchfelder beispielsweise in Form von technologischen und nachhaltigkeitsrelevanten Fragestellungen zu strukturieren und deren Bewertung durch die Analyse und Beobachtung von Einflussfaktoren $\mathrm{zu}$ ermöglichen. Dabei spielt die Identifikation und Nutzung von Experten und ihrem Fachwissen eine entscheidende Rolle.

Unterstützt wird die Vorgehensweise durch die im Forschungsprojekt entwickelten Softwaretools Semafor und Expose, die den Arbeitsaufwand für die genannten Aufgaben deutlich reduzieren. In ihrer Kombination liefern die beiden
Softwaretools eine gute Unterstützung bei der Entscheidungsfindung in frühen Innovationsphasen.

\section{Anmerkungen}

(1) Weitere Informationen sind auf der Webseite des Projektes www.novanet.de verfügbar.

\section{Literatur}

Angrick, M. (Hrsg.): Auf dem Weg zur nachhaltigen Informationsgesellschaft. Marburg 2003.

Fichter, K.: Interpreneurship, Nachhaltigkeitsinnovationen in interaktiven Perspektiven unternehmerischen Handelns. Marburg 2005.

Fichter, K. / Beucker, S.: Wandel der Innovationsbedingungen in der Internetökonomie - Erklärungsbedürftige Phänomene im Themenfeld Innovation und Internetökonomie. Nova-net Werkstattreihe, Stuttgart 2006.

Fichter, K. / Noack, T. / Beucker, S. / Bierter, W. / Springer, S.: Nachhaltigkeitskonzepte für Innovationsprozess. Nova-net Werkstattreihe, Stuttgart 2005.

Jakob, M. / Kiehne, D.-O / Schwarz, H. / Kaiser, F. / Beucker, S.: Delphigestütztes Szenario-Management und -Monitoring. Nova-net Werkstattreihe, Stuttgart 2007.

Jakob, M. / Kaiser, F. / Schwarz, H.: SEMAFOR: A Framework for an Extensible Scenario Management System. In: Proc. of the IEEE International Engineering Management Conference (IEMC) 2005 in St. John's, Newfoundland, September 11-14, 2005.

Kaiser, F. / Schwarz, H. / Jakob, Mihály: EXPOSE: Searching the Web for Expertise. Demo. In: Proceedings of the 30th Annual International ACM SIGIR Conference on Research and Development in Information Retrieval, Amsterdam, The Netherlands, July 23-27, 2007.

Müller-Stewens, G. / Lechner, C.: Strategisches Management: Wie strategische Initiativen zum Wandel führen - der St. Galler General Management Navigator. Stuttgart 2001

Paech, N. / Pfriem, R.: Konzepte der Nachhaltigkeit von Unternehmen. Theoretische Anforderungen und empirische Trends. Schriftenreihe am Lehrstuhl für Allg. BWL, Unternehmensführung und Betriebliche Umweltpolitik Nr. 37/2004, Carl-von-Ossietzky-Universität Oldenburg.

Züst, R.: Produkte im Fokus des Umweltmanagements. In: Winter., G.: Das umweltbewusste Unternehmen. München 1998.

\section{【 AUTOREN + KONTAKT}

Severin Beucker ist Mitgründer und Senior Researcher am Borderstep Institut. E-Mail: beucker@borderstep.de

Fabian Kaiser arbeitet als wissenschaftlicher Mitarbeiter am Institut für Parallele und Verteilte Systeme (IPVS) der Universität Stuttgart.

E-Mail: fabian.kaiser@ipvs.uni-stuttgart.de

Mihály Jakob ist wissenschaftlicher Mitarbeiter am Institut für Parallele und Verteilte Systeme, Abteilung Anwendersoftware der Universität Stuttgart. E-Mail: mihaly.jakob@ipvs.uni-stuttgart.de.

Dierk-Oliver Kiehne ist Mitgründer und geschäftsführender Gesellschafter der InTraCoM GmbH. E-Mail: info@intracomgroup.de
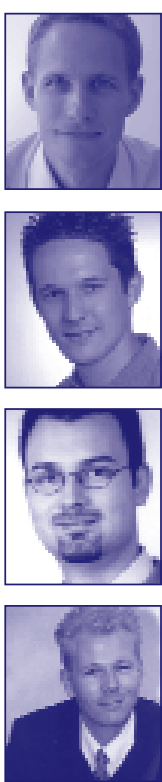
(c) 20I0 Authors; licensee IÖW and oekom verlag. This is an article distributed under the terms of the Creative Commons Attribution Non-Commercial No Derivates License (http://creativecommons.org/licenses/by-nc-nd/3.o/), which permits unrestricted use, distribution, and reproduction in any medium, provided the original work is properly cited. 